\title{
Identification and characterization of key circadian clock genes of tobacco hairy roots: putative regulatory role in xenobiotic metabolism
}

Lucas G. Sosa Alderete, $1,2 \square$

Phone +54 3584676537

Emaillucasaureus@gmail.com

Emaillsosa@exa.unrc.edu.ar

Mario E. Guido, 3

Emailmguido@fcq.unc.edu.ar

Elizabeth Agostini, 1,2

Email eagostini@exa.unrc.edu.ar

Paloma Mas, 4,5

Email paloma.mas@cragenomica.es

${ }^{1}$ Department of Molecular Biology, UNRC, Río Cuarto, Argentina AQ1

2 Consejo Nacional de Investigaciones Científicas y Técnicas (CONICET), Córdoba, Argentina

3 CIQUIBIC-CONICET, UNC, Córdoba, Argentina

${ }^{4}$ Center for Research in Agricultural Genomics (CRAG), Barcelona, Spain

5 Consejo Superior de Investigaciones Científicas (CSIC), Barcelona, Spain

Received: 6 June 2017 / Accepted: 24 October 2017

\section{Abstract}

The circadian clock is an endogenous system that allows organisms to daily adapt and optimize their physiology and metabolism. We studied the key circadian clock gene (CCG) orthologs in Nicotiana tabacum seedlings and in hairy root cultures (HRC). Putative genes involved in the metabolism of 
xenobiotic compounds (MXC) were selected and their expression profiles were also analyzed. Seedlings and HRC displayed similar diurnal variations in the expression profiles for the CCG examined under control conditions (CC). MXC-related genes also showed daily fluctuations with specific peaks of expression. However, when HRC were under phenol treatment (PT), the expression patterns of the clock and MXC-related genes were significantly affected. In 2-week-old HRC, PT downregulated the expression of NtLHY, $N t T O C 1$, and NtPRR9 while NtFKF1 and NtGI genes were upregulated by phenol. In 3-week-old HRC, PT also downregulated the expression of all CCG analyzed and NtTOC1 was the most affected. Following PT, the expression of the MXC-related genes was upregulated or displayed an anti-phasic expression profile compared to the expression under CC. Our studies thus provide a glimpse of the circadian expression of clock genes in tobacco and the use of HRC as a convenient system to study plant responses to xenobiotic stresses.

\section{Keywords}

Tobacco hairy roots

Circadian clock

Phenol treatment

Xenobiotic metabolism

Responsible editor: Philippe Garrigues

Electronic supplementary material

The online version of this article ( https://doi.org/10.1007/s11356-017-0579-9) contains supplementary material, which is available to authorized users.

\section{Introduction}

Plants are constantly exposed to environmental fluctuations affecting their growth and development and, as consequence, crop productivity is negatively impacted. Drought, high salinity, extreme temperature and heavy metals are among the most common abiotic stress factors. However, in the last decades, the indiscriminate use of agrochemicals and the release of pollutants, such as phenols, into soils have created another stressful condition for plant growth. In this context, plants have developed numerous strategies to quickly detect these adverse growth conditions, triggering a series of morphological, physiological, biochemical, and molecular changes that affect their growth but also improve their capacity to adaptation and survival (Harb et al. 2010). 
The circadian clock plays a key role in plant adaptation to stress through synchronization of metabolism and physiology with the environmental changes (Kant et al. 2008; Dong et al. 2011; Sanchez, et al. 2011; Seo and Mas 2015). Thus, it was proposed that a major function of the circadian clock is to confer an adaptive advantage (Dodd et al. 2005) as reviewed in Vaze and Sharma (2013).

Mechanistically, the daily changes of light and temperature act as synchronizing signals to entrain every day the oscillator, which generates the rhythms in multiple biological processes or clock outputs. A number of different clock components with specific peak-phases of expression have been described in Arabidopsis thaliana as reviewed in McClung (2006). The complex regulatory networks among these components precisely define the 24-h rhythms in their expression. Genes expressed during the day include, for instance two singleMYB transcription factors, CIRCADIAN CLOCK ASSOCIATED1 (CCA1), LATE ELONGATED HYPOCOTYL (LHY) (Alabadi et al. 2002; Harmer and Kay 2005; Yakir et al. 2009; Lu et al. 2009), and the PSEUDO-RESPONSE REGULATOR (PRR) genes, such as PRR9 and PRR7 (Salomé and McClung 2005; Farre et al. 2005; Nakamichi et al. 2007). Another member of the PRR family, PRR1 or TOC1 (TIMING OF CAB EXPRESSION1) exhibit a peak of expression close to dusk (Alabadi et al. 2001) while EARLY FLOWERING 3 (ELF3), EARLY FLOWERING 4 (ELF4) and LUX ARRHYTHMO (LUX), components of the evening complex (EC), have a peak of expression during the night (Nagel and Kay 2012). TOC1 protein stability is tightly controlled by the F-box protein ZEITLUPE (ZTL) (Mas et al. 2003) which physically interacts with GIGANTEA (GI), an evening-expressed clock component (Mishra and Panigrahi 2015). GI also interact with FLAVIN-BINDING, KELCH REPEAT, F-BOX1 (FKF1) and this $G I-F K F 1$ complex mediates the ubiquitin-dependent degradation of CYCLING DOF FACTOR (CDF) proteins that represses the transcription of genes involved in flowering time such as CONSTANS (CO) and FLOWERING LOCUS T (FT) genes (Sawa et al. 2007; Song et al. 2014). Although, the majority of information available about the circadian clock suggests conservation among plant species, the circadian function in other plants different than Arabidopsis still remains to be fully elucidated. N. tabacum (tobacco) is one of the most commercially important crop plants. Some orthologs to the Arabidopsis circadian clock genes, with conserved oscillatory behavior in their expressions, have been identified in Nicotiana (Yon et al. 2012). However, the information about the circadian clock function in $N$. tabacum remains very scarce.

Regarding the use of hairy roots cultures (HRC) for circadian rhythm studies, there is only one report performed in Datura innoxia HRC (Lanoue et al. 2004). 
The authors showed that under controlled conditions, $\mathrm{pH}$ and conductivity signals detected in culture medium exhibited circadian oscillations. Till now, HRC from different plant species such as $N$. tabacum have been used mainly to study different physiological, biochemical, and molecular process related to the phytoremediation of environmental pollutants such as phenol. These studies have demonstrated that peroxidases have an active participation in the phenol removal process (Agostini et al. 2003; Coniglio et al. 2008; Sosa Alderete et al. 2009; Talano et al. 2010; Sosa Alderete et al. 2011; Ibaáñez et al. 2012). In this sense, it is well known that plants can detoxify or store organic compounds by using detoxification mechanisms similar to that of the mammalian hepatic system and thus the term green liver was adopted (Abhilash et al. 2009). This detoxification model includes three common steps such as (I) initial transformation where oxidation reactions are predominant and can be catalyzed mainly by cytochrome P450 and peroxidases, (II) conjugation step involving the addition of D-glucose or gluthatione by catalytic action of transferases such as glucosyl transferase (GT) or glutathione S-transferase (GsT), and (III) sequestration step, where conjugated compounds are stored in vacuole or cell wall. In mammals, it is known that enzymes belonging to I, II, and III phases of metabolism of xenobiotic compounds (MXC) have a strong circadian regulation (Zmrzljak and Rozman 2012). However, in plants, there is no information about the putative circadian regulation on these genes or their products involved in MXC. Thus, here, we address these studies by (i) investigating if HRC were able to sustain the $24 \mathrm{~h}$ oscillations in expression of key clock genes, (ii) examining the effect of phenol treatment on clock gene expression, (iii) analyzing the putative circadian regulation of genes involved in MXC such as cytochrome monooxygenase (CYP71D21), peroxidase (NtPXC8), glycosyl transferase $(N t G T)$, glutathione S-transferase ( $N t G s T$ ) belonging to phase I and II of MCX, respectively.

\section{Material and methods}

\section{Biological material}

Tobacco (N. tabacum var. Wisconsin) seedlings and HRC employed for the experiments were maintained in a grown chamber under constant conditions of light $\left(60-100 \mu \mathrm{mol} \mathrm{m} \mathrm{m}^{-2} \mathrm{~s}^{-1}\right.$ of cool white fluorescent light) and temperature $\left(24 \pm 2{ }^{\circ} \mathrm{C}\right)$ for 14 and 21 days on MS medium enriched with vitamins (Murashige and Skoog 1962). HRC were maintained according to conditions previously described by Sosa Alderete et al. (2009). Tobacco seedling was grown in solid MS medium for 21 days while HRC were grown either in liquid and solid MS medium for 14 and 21 days. HRC growing in liquid medium were with an orbital shaker at $70 \mathrm{rpm}$. 


\section{Seedlings/HRC synchronization and sampling}

Tobacco seedlings and HRC were synchronized by light/dark $(12 \mathrm{~h} \mathrm{light} / 12 \mathrm{~h}$ dark) cycles $\left(60-100 \mu \mathrm{mol} \mathrm{m} \mathrm{m}^{-2} \mathrm{~s}^{-1}\right.$ of cool white fluorescent light) at $24 \pm 2{ }^{\circ} \mathrm{C}$ for at least 7 days. Tobacco seedlings and HRC were harvested in duplicate or triplicate every $4 \mathrm{~h}$ for at least $24 \mathrm{~h}$. Then, samples were dried with filter paper and grounded to powder using liquid $\mathrm{N}_{2}$ and stored at $-80{ }^{\circ} \mathrm{C}$ for further studies, such as RNA extraction and gene expression analysis.

\section{Phenol treatment assay}

Inocula of $0.4 \mathrm{~g}$ of $\mathrm{HRC}$ were placed in Erlenmeyer flasks containing $50 \mathrm{ml}$ of MS medium and incubated at $24 \pm 2{ }^{\circ} \mathrm{C}$ on an orbital shaker at $70 \mathrm{rpm}$. On the one hand, 2-week-old HRC were grown during the first week under darkness condition while from the second week to the end of experiment, HRC were entrained by light/dark ( $12 \mathrm{~h}$ light $/ 12 \mathrm{~h}$ dark) cycles $\left(60-100 \mu \mathrm{mol} \mathrm{m}^{-2} \mathrm{~s}^{-1}\right.$ of cool white fluorescent light) at $24 \pm 2{ }^{\circ} \mathrm{C}$ for at least 7 days. On the other hand, 3 -week-old HRC were grown under darkness condition in the first 2 weeks while from the third week to the end of experiment, HRC were entrained by light/dark cycles (Fig. S1). After 2 or 3 weeks of growth, HRC were treated, in the light phase (ZT 3), with phenol (100 mg/L) during $24 \mathrm{~h}$. For that, a volume of phenol from a stock solution, previously sterilized by filtration using $0.22-\mu \mathrm{m}$ Millipore filters was added to the growth medium. As control conditions, instead of phenol $1 \mathrm{~mL}$ of distilled sterilized water was added to the culture medium. Then, HRC were harvested at each 4-h intervals from the time the lights came on (Zeitgeiber time (ZT) 0), for at least $24 \mathrm{~h}$ and immediately frozen as described above, for further assays.

\section{Quantitative-PCR analysis of genes expression}

Gene expression was evaluated in seedlings only under control condition and in HRC subjected to phenol treatment by quantitative real-time PCR (qPCR). All of the experiments were completed with three biological replicates $(n=3)$, collected, and pooled. Each replicate tissue set was ground to a fine powder in liquid $\mathrm{N}_{2}$, and total RNA was isolated using Trizol Reagent (Invitrogen) according to the manufacturer's instructions. The yield and purity of RNA were estimated by optical density at $260 / 280 \mathrm{~nm}$. Total RNA $(1 \mu \mathrm{g})$ was treated with DNase (Promega) during $30 \mathrm{~min}$ at $37^{\circ} \mathrm{C}$. Then, for the cDNA synthesis, MMLV reverse transcriptase (Epicentre) and random hexamer primers (Biodynamics) were added in the same tube at final volume of $25 \mu 1$ according to the manufacturer's indications. The amplification mix contained $1 \mu 1$ of the cDNA, $400 \mathrm{nM}$ forward-reverse primers, and $7.5 \mu \mathrm{l}$ of Itaq Universal SYBR Green supermix $2 \times$ (Biorad) in a total volume of $15 \mu 1$. The cycling conditions 
were $3 \mathrm{~min}$ at $95.0^{\circ} \mathrm{C}$, and 40 cycles of $95.0^{\circ} \mathrm{C}$ for $15 \mathrm{~s}, 60.0^{\circ} \mathrm{C}$ for $30 \mathrm{~s}$ and $72{ }^{\circ} \mathrm{C}$ for $30 \mathrm{~s}$. Each qPCR was performed in duplicate for each independent biological replicates $(n=3)$. The standard curve linearity and PCR efficiency $(E)$ were optimized for each pair of primers. Although $N$. tabacum specie is an allotetraploid, it is important to note that all primers employed in this work were specific to a single gene. The normalized expression (NE) was calculated using the following formula: $\mathrm{NE}=2^{-\Delta \mathrm{Ct}}$, where $\Delta \mathrm{Ct}=\mathrm{Ct}$ experimental $-\mathrm{Ct}$ normalizer. Elongation factor 1 (NtEFl) gene was used as reference gene, previously identified as stably expressed under many developmental stages and stress situations (Schmidt and Delaney 2010). The primers for the NTLHY, NTTOC1 and NTFKF1 clock genes were previously described in Yon et al. (2012), whereas the primers for the NTGI and NTPRR9 and putative genes involved in MXC (NTPXC8.1, CYP71D21, GT and GsT) were designed using N. tabacum ortholog sequences obtained from Sol Genomics Network (SGN) ( http://solgenomics.net ) or National Center of Biotechnology information (NCBI) ( https://www.ncbi.nlm.nih.gov ) databases. Then, the selected tobacco gene sequences were used to design specific primers to a single gene by using Integrated DNA technologies tools ( https://www.idtdna.com ) (Primers list is shown in Supporting information Table S1). The putative genes involved in MXC were selected according to previous works that confirmed the upregulation of these genes under stress conditions (Takahashi and Nagata 1992; FraissinetTachet et al. 1998; Ralston et al. 2001).

\section{Identification of $N$. tabacum circadian clock genes}

To identify homolog circadian clock genes of Arabidopsis in the N. tabacum genome, the corresponding amino acid sequences of Arabidopsis obtained from TAIR ( https://www.arabidopsis.org ) database were used as queries in BLAST searches (TBLASTN tool) against the $N$. tabacum genome TN90 through the use of the SGN and NCBI databases. The most similar tobacco nucleotide sequences were selected based on whether they had $e$ values close to 0 (Supporting information Table S2).

\section{Identification of cis elements in the promoter regions of putative MXC-involved genes under circadian control}

The cis-regulatory elements in the promoter regions of tobacco genes were identified using the PlantCARE (plant cis-acting regulatory elements) tools (Lescot et al. 2002). To this, the promoter regions of tobacco genes including $1.5 \mathrm{~kb}$ sequences upstream of the transcription start site were analyzed (Supporting information Table S3). Subsequently, homologs of tobacco genes involved in MXC were identified in A. thaliana by using predicted nucleotide sequences of a given tobacco genes as a query in BLASTX searches using NCBI 
and the TAIR databases. The most similar Arabidopsis amino acid sequences were selected based on $e$ values and score (Supporting information Table S4). Then, promoter regions of $A$. thaliana putative genes involved in MXC including $1.5 \mathrm{~kb}$ sequences upstream of the transcription start site were used for search of cis-regulatory elements by using of AGRIS (Arabidopsis gene regulatory information) server ( http://arabidopsis.med.ohiostate.edu/AtcisDB/atcisview.html ) (Supporting information Table S5).

\section{Data analysis}

Results were obtained from at least three independent biological replicates where each one was evaluated in two technical replicates. Then the data were statistically processed by analysis of variance (ANOVA) using the STATISTICA 6.0 software and applying the Duncan test $(p<0.05)$. For the normalization of relative gene expression, $N t E F 1$ gene was used as an internal control.

\section{Results and discussion}

\section{Identification and characterization of key circadian clock genes in $N$. tabacum seedlings}

We analyzed the expression of several genes in $N$. tabacum such as NtLHY, NtTOC1, NtFKF1, NtGI and NtPRR9, which are orthologs of A. thaliana clock genes, previously described (Más 2008). To that end, we first used 3-week-old seedlings maintained under a $12 \mathrm{~h} \mathrm{~L} / 12 \mathrm{~h}$ D cycle. The circadian clock genes analyzed showed a rhythmic pattern of expression. $N t L H Y$ displayed a peak expression closed to dawn (Fig. 1a) while NtFKF1 and NtTOC1 showed a peak expression around Zeitgeber time 12 (ZT12), i.e. closed to dusk (Fig. 1b, c). To identify and characterize $N t G I$ and $N t P R R 9$ gene expression in N. tabacum, a Blast tool was performed using as query both orthologous clock genes from $A$. thaliana, which were run against whole tobacco genome available in the SGN database. The putative clock genes (NtGI and NtPRR9), found in the tobacco genome, were selected based on the highest homology and identity (over 80\%) and the lowest $e$ values. Gene expression analyses showed that NTGI and $N t P R R 9$ also diurnally oscillated with peaks of expression during the light phase (around ZT 7 for NtGI and ZT 3-ZT 7 for NtPRR9) (Fig. 1d, e). These results showed that all the tobacco clock genes examined displayed a rhythmic oscillation with a similar peak phase to that described for the ortologs in $A$. thaliana (Más 2008) and in N. attenuate (Yon et al. 2012).

\section{Fig. 1}

Analysis of clock gene expression: $N t \mathrm{LHY}(\mathbf{a}), N t \mathrm{TOC} 1$ (b), $N t \mathrm{FKF} 1(\mathbf{c}), N t \mathrm{GI}$ (d) and NtPRR9 (e) by q-RT PCR. N. tabacum seedlings were maintained in growth 
chamber during 3 weeks in solid MS medium and synchronized by light/dark ( $12 \mathrm{~h}$ light/12 h dark) cycles at $24 \pm 2{ }^{\circ} \mathrm{C}$ for at least 7 days. White and black bars represents light and dark phases, respectively. Data are expressed as the mean of $n=3$ biological replicates \pm SEM (standard error of the mean). Relative expression was normalized using elongation factor $1(N t E F l)$ as housekeeping gene
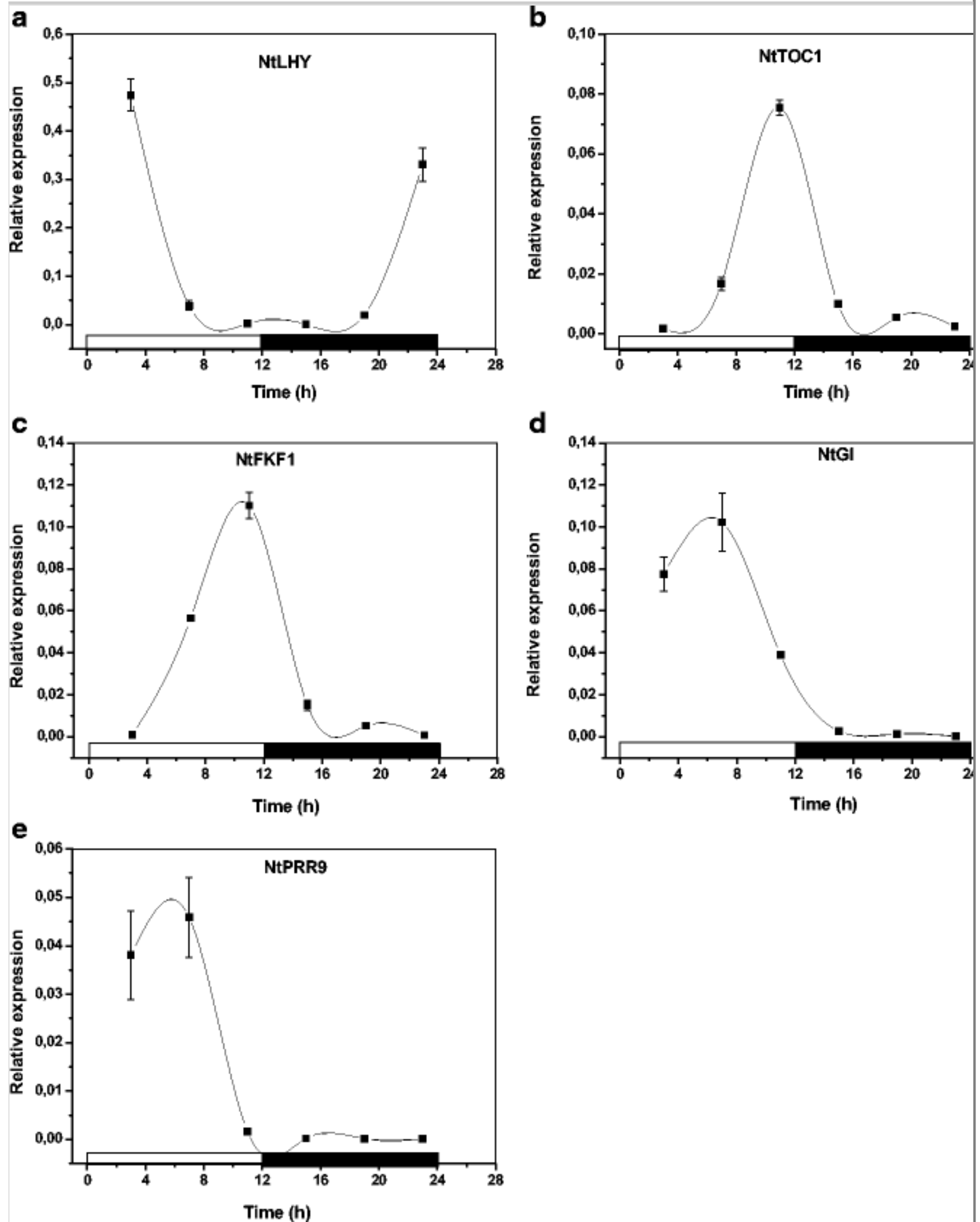


\section{Effects of phenol treatment on clock gene expression in tobacco hairy roots}

Next, we evaluated the circadian clock gene expression in HRC. These cultures usually grow under darkness with or without orbital agitation. Thus, we first explored whether the exposition to photoperiodic conditions would synchronize the circadian expression of clock genes in HRC. Our results indeed showed that all clock genes examined displayed a rhythmic pattern of expression (Fig. 2) similar to that observed in intact seedlings. In order to determine the possible effects of the culture medium (Murashige and Skoog, MS, solid and liquid) and age of cultures on clock gene expression, HRC were grown during 2 and 3 weeks both in liquid as well as in solid medium. The results showed that the expression profiles of clock genes were similar to those obtained with seedlings regardless the HRC age or the culture medium. NtTOCl and NtFKF1 clock genes maintained their peaks at ZT 11 (Figs. 2, 3b, c) while $N t L H Y$ peak expression was around dawn (Figs. 2, 3a). Regarding NtGI and NtPRR9, both genes maintained a similar rhythmic expression compared to tobacco seedlings, with peaks around ZT 7 and ZT 3, respectively (Figs. 2, 3d, e). HRC grown in solid MS medium showed the same temporal expression profiles as those grown in liquid MS medium or tobacco seedlings (Supporting information Fig. S2). To examine whether the oscillatory expression is truly circadian, we examined rhythms in HRC maintained under constant dark conditions (free running) during at least 7 days. Our results showed a clear rhythmic pattern in the expression of some $N t$ clock genes in 2-week-old HRC (Supporting information Fig. S3a), suggesting that the circadian clock controls indeed their expression. However, in 3-week-old HRC, the clock genes seem to loss their rhythmic expression patterns since not significant changes in the expression values were found (Supporting information Fig. S3b).

\section{Fig. 2}

Analysis of clock gene expression: $N t \mathrm{LHY}(\mathbf{a}), N t \mathrm{TOC} 1$ (b), $N t \mathrm{FKF} 1(\mathbf{c}), N t \mathrm{GI}$ (d) and NtPRR9 (e) by q-RT PCR. N. tabacum hairy root cultures (HRC) were maintained in growth chamber during 2 weeks in liquid MS medium with orbital shaking at $70 \mathrm{rpm}$ and synchronized by light/dark ( $12 \mathrm{~h}$ light/12 $\mathrm{h}$ dark) cycles at $24 \pm 2{ }^{\circ} \mathrm{C}$ for at least 7 days. Data are expressed as the mean of $n=3$ biological replicates \pm SEM (standard error of the mean). White and black bars represents light and dark phases, respectively. Relative expression was normalized using elongation factor 1 (NtEF1) as housekeeping gene. Black and grey lines represent HRC treated with water (control condition) and phenol (100 mg/L during $24 \mathrm{~h}$ ), respectively 

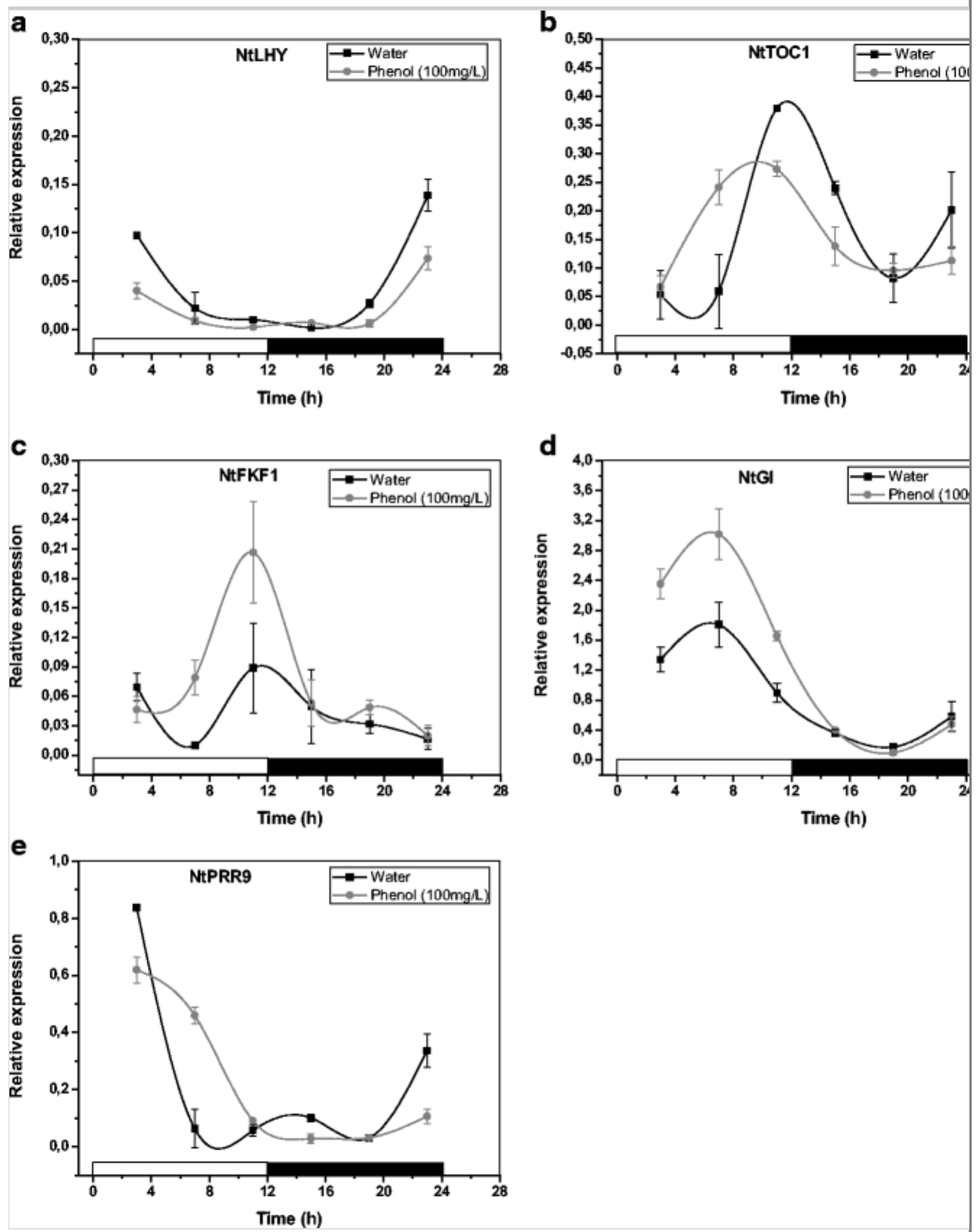

Fig. 3

Analysis of clock gene expression: $N t \mathrm{LHY}(\mathbf{a}), N t \mathrm{TOC} 1$ (b), $N t \mathrm{FKF} 1(\mathbf{c}), N t \mathrm{GI}$ (d) and NtPRR9 (e) by q-RT PCR. $N$. tabacum hairy root cultures were maintained in growth chamber during 3 weeks in liquid MS medium with orbital shaking at $70 \mathrm{rpm}$ and synchronized by light/dark (12 h light/12 h dark) cycles at $24 \pm 2{ }^{\circ} \mathrm{C}$ 
for at least 7 days. Data are expressed as the mean of $n=3$ biological replicates \pm SEM (standard error of the mean). White and black bars represent light and dark phases, respectively. Relative expression was normalized using elongation factor 1 $(N t E F 1)$ as housekeeping gene. Black and grey lines represent HRC treated with water (control condition) and phenol (100 mg/L during $24 \mathrm{~h})$, respectively
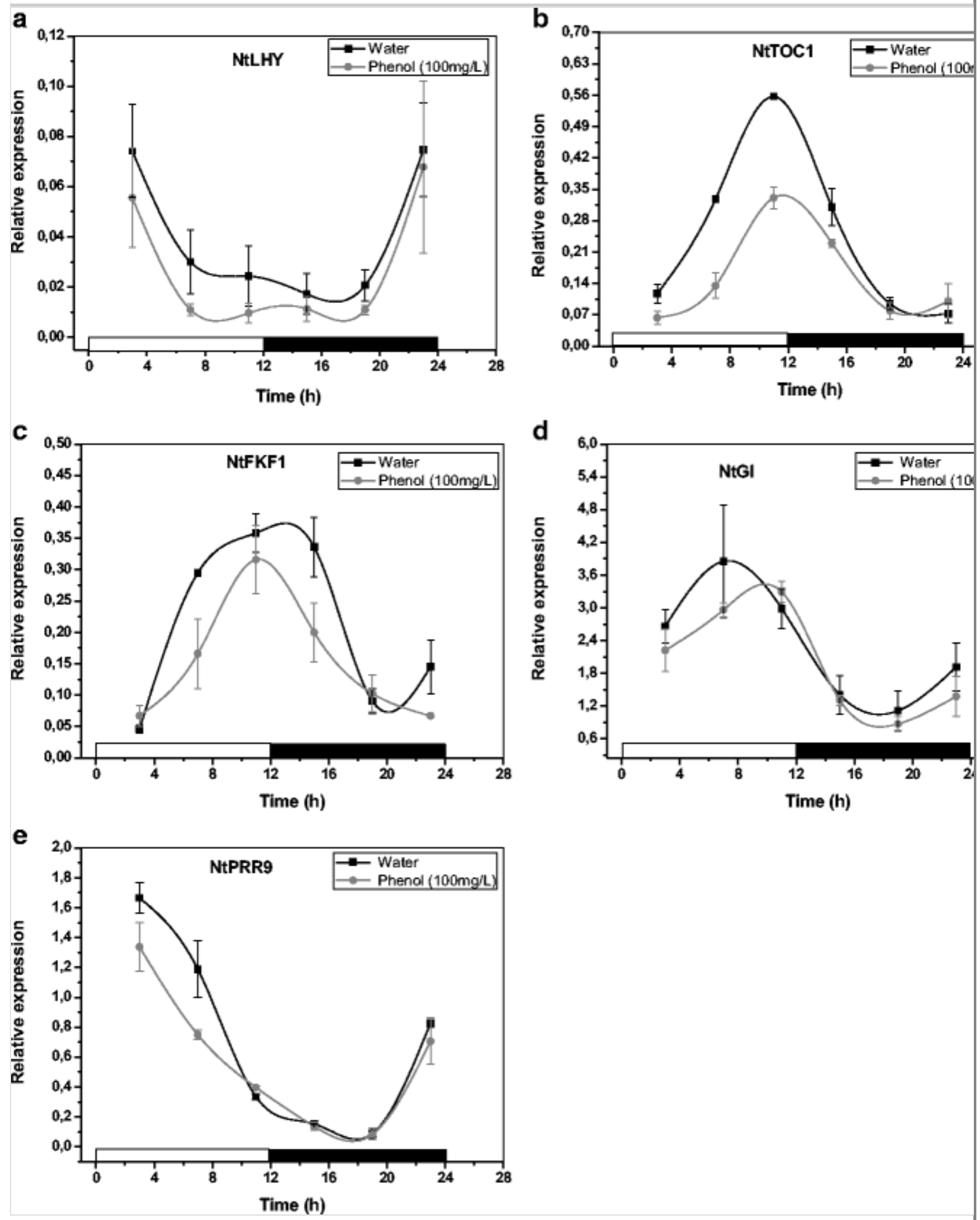
We next examined the expression of circadian clock genes following stress by an organic pollutant exposure such as phenol. HRC grown in liquid MS medium for 2 or 3 weeks were exposed to phenol $(100 \mathrm{mg} / \mathrm{L})$ for $24 \mathrm{~h}$ and clock gene expression was evaluated. In HRC of 2 weeks, phenol treatment downregulated the expression of several clock genes including NtLHY, NtTOC1 and NtPRR9 (Fig. 2a, b, e). A phase advanced was also observed for NtTOCl. Similar findings were described by Marcolino-Gomes et al. (2014) who demonstrated that soybean plants exposed to severe drought stress not only advanced the phase of TOC1 gene but also downregulated the expression of circadian clock components. Contrarily, phenol treatment significantly upregulated, almost twofold, the expression of NtFKF1 and NtGI genes (Fig. 2c, d). These results are also consistent with those observed in barley roots exposed to osmotic stress, showing an upregulation in the expression of several clock genes (Habte et al. 2014).

In 3-week-old HRC, phenol exposure induced a significant downregulation in the expression of all clock genes studied (NtLHY, NtTOC1, NtFKF1, NtGI and $N t P R R 9$ ) as compared with water-treated controls (Fig. 3a-e). In particular, NtTOC1 expression was the most affected by phenol since its expression levels showed higher differences than its control (Fig. 3b). Treatment at this stage did not show changes in NtTOC1 phase as observed in 2-week-old HRC. In contrast, phenol treatment seemed to delay the phase of $N t G I$ (Fig. 3d).

Together, these results represent to our knowledge, the first evidence at a molecular level, about circadian gene expression in N. tabacum HRC. Our data also shows that the circadian expression is modulated by phenol treatment, which opens the question about a role for the circadian clock in plant responses to phenol exposure.

\section{Identification and circadian characterization of putative genes involved in metabolism of xenobiotic compounds}

Later we performed a selection of putative genes encoding for enzymes involved in the phase I and II of metabolism of xenobiotic compounds (MCX), such as a class III peroxidase (NtPXC8.1), a cytochrome P450 (NtCYP71D21), a glycosyl transferase $(N t G T)$, a glutathione S-transferase $(N t G s T)$, respectively. These genes would encode for enzymes involved in phenolics detoxification pathways. Firstly, we evaluated the oscillatory expression profiles in tobacco seedlings grown under $12 \mathrm{~h} \mathrm{~L} / 12 \mathrm{~h} \mathrm{D}$ cycles. CYP71D21 and NtPXC8.1 genes showed their peak phases during the night, around ZT 15 and 19, respectively (Fig. 4a, b). NtGsT and $N t G T$ genes showed clear differences in their expression profiles, 
since $N t G s T$ picked before dusk (around ZT 11) whereas the peak expression of NtGT gene was around ZT 15-ZT 19 (dark phase) (Fig. 4c, d).

\section{Fig. 4}

Expression analysis of putative genes involved in metabolism of xenobiotic compounds: $N t C Y P 71 D 21$ (a), NtPXC8.1 (b), NtGsT (c) and NtGT (d) by q-RT PCR. N. tabacum seedlings were maintained in growth chamber during 3 weeks in solid MS medium and synchronized by light/dark (12 h light/12 h dark) cycles at $24 \pm 2{ }^{\circ} \mathrm{C}$ for at least 7 days. Data are expressed as the mean of $n=3$ biological replicates \pm SEM (standard error of the mean). White and black bars represent light and dark phases, respectively. Relative expression was normalized using elongation factor 1 (NtEFl) as housekeeping gene
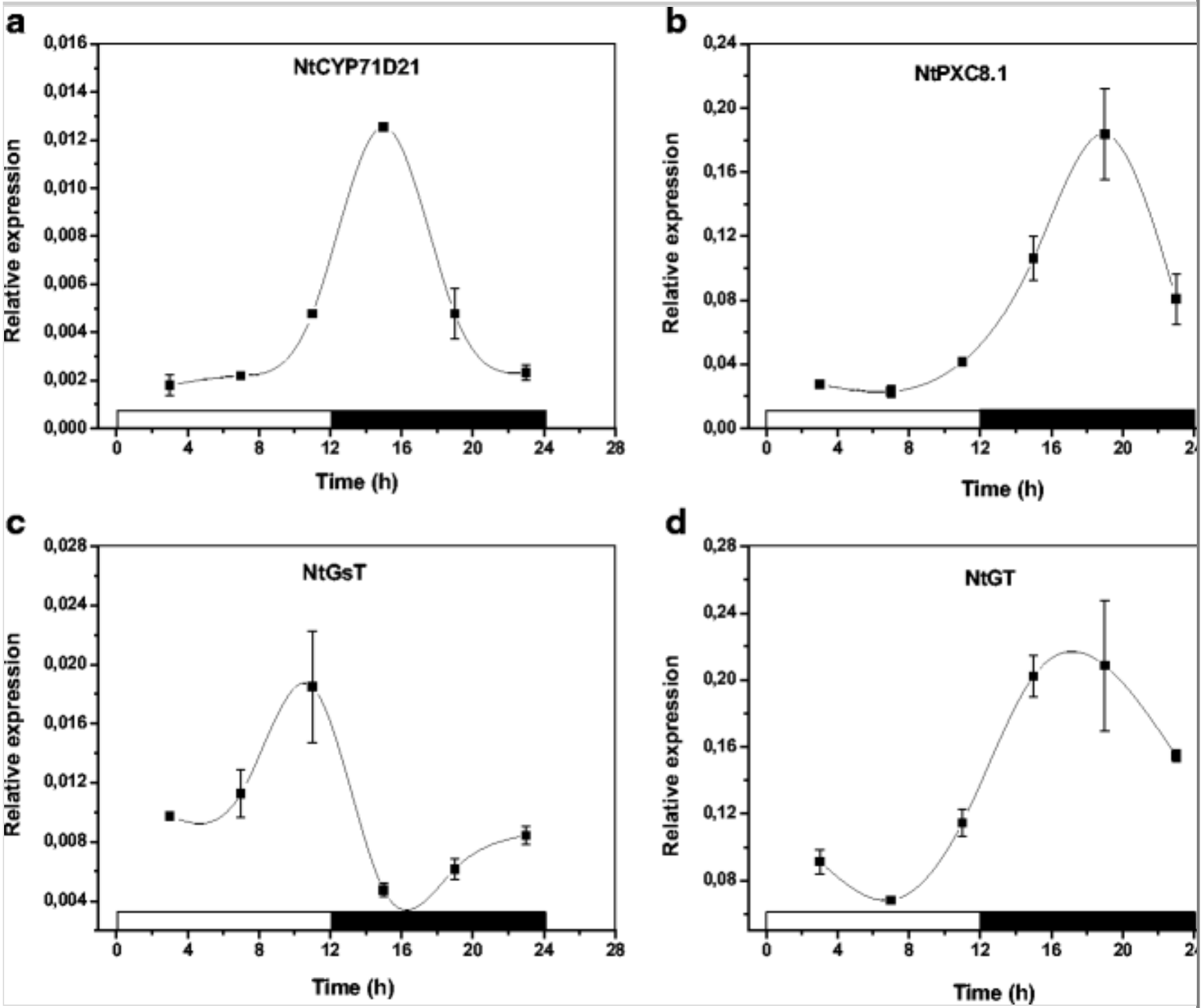

Remarkably, three of the four MCX-related genes examined (CYP71D21, $N t P X C 8.1$ and $N t G T$ ) display a clear nocturnal peak likely revealing a higher requirement of redox enzyme activity and detoxification during the dark phase. Cytochrome P450 (CYP71D21) is a key enzyme in the transformation of several 
xenobiotic compounds, since oxidations by P450 enzyme families are predominant detoxification mechanisms for many plants. However, another important enzyme family, like peroxidases (PX), plays a significant role in the transformation step since they may oxidize phenolic compounds while consuming hydrogen peroxide (Abhilash et al. 2009; Agostini et al. 2013). Following transformation, conjugation is predominantly the next detoxification step of xenobiotic compounds and the resulting conjugates can be deposited in vacuoles or incorporated into bound residues through sequestration. Although, some compounds are conjugated without being preceded by transformation, other xenobiotic compounds are previously transformed forming functional groups necessary for the conjugation with different molecules such as D-glucose or glutathione. These reactions are catalyzed by glycolsyltransferase (GT) or gluthatione S-transferase (GST), respectively. Our results showed that the mRNA for the glycosyltransferase (NtGT) peaked also in the dark phase. Therefore, it is probably that phenol glycosilation catalyzed by NtGT might be active also during the night phase, due to these mechanisms usually take place simultaneously. In this sense, previous reports have described an increase in Oglucosyltransferase activities for detoxification of chlorinated phenols in different plant species and plant cell suspension cells (Burken, 2003). Regarding the activities of GST, they are also considered to be one of the primary inactivation mechanisms for toxic chemicals and have been shown to be important in response to oxidative stress. These enzymes are ubiquitous in plants and active toward a broad range of xenobiotic substrates (Burken, 2003). Results found in this work showed an increase in expression of the glutathione Stransferase (NtGsT) at the end of the light phase. Thus, we suggest this protein might exhibit the ability not only to catalyze the conjugation of glutathione (GSH) to xenobiotic substrates for the purpose of detoxification but also to display an active participation in response to the oxidative stress induced by phenol exposure at the end of the light phase.

$\mathrm{AQ} 2$

When the putative genes involved in MXC were analyzed in HRC of 2 and 3 weeks of growth, the genes showed characteristic expression profiles under control conditions. In 2-week-old HRC under control conditions, NtCYP71D21 and NtPXC8.1 genes showed a similar expression pattern compared to tobacco seedlings, with highest expression values around ZT 15 and ZT 19, respectively (Fig. 5a, b). However, $N t G s T$ and $N t G T$ genes did not show a robust oscillatory behavior in their expression profiles due to the absence of clear circadian waveforms (Fig. 5c, d). In 3-week-old HRC, $N t G s T$ and $N t G T$ genes showed expression profiles similar to the ones observed in seedlings, with their highest expression at ZT 11 and ZT 15, respectively (Fig. 6c, d). NtPXC8.1 showed a decreased amplitude and an advance phase of approximately $4 \mathrm{~h}$ (peak phase 
around ZT 15) in comparison with the peak in seedlings or in 2-week-old HRC where the peak was at ZT 19 (Figs. 4, 5b). NtCYP71D21 showed a nonoscillatory profile during the diurnal cycle (Fig. 6a).

Fig. 5

Expression analysis of putative genes involved in metabolism of xenobiotic compounds: NtCYP71D21 (a), NtPXC8.1 (b), NtGsT (c) and NtGT (d) by q-RT PCR. N. tabacum hairy root cultures (HRC) were maintained in growth chamber during 2 weeks in liquid MS medium with orbital shaking at $70 \mathrm{rpm}$, and synchronized by light/dark (12 h light/12 h dark) cycles at $24 \pm 2{ }^{\circ} \mathrm{C}$ for at least 7 days. Data are expressed as the mean of $n=3$ biological replicates \pm SEM (standard error of the mean). White and black bars represent light and dark phases, respectively. Relative expression was normalized using elongation factor 1 (NtEFl) as housekeeping gene. Black and grey lines represent HRC treated with water (control condition) and phenol (100 mg/L during $24 \mathrm{~h}$ ), respectively
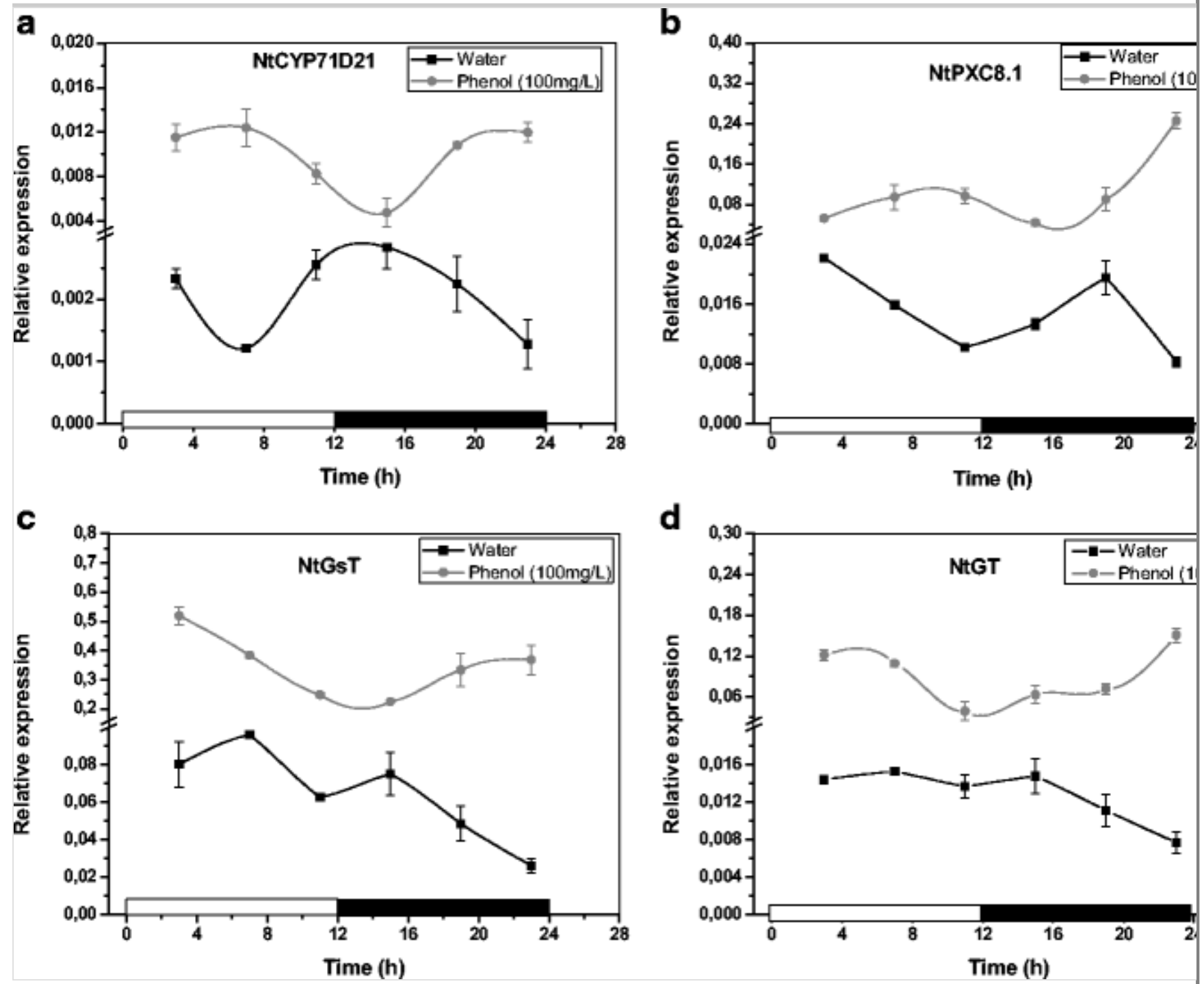

Fig. 6 
Expression analysis of putative genes involved in metabolism of xenobiotic compounds: NtCYP71D21 (a), NtPXC8.1 (b), NtGsT (c) and NtGT (d) by q-RT PCR. N. tabacum hairy root cultures (HRC) were maintained in growth chamber during 3 weeks in liquid MS medium with orbital shaking at $70 \mathrm{rpm}$, and synchronized by light/dark (12 h light/12 h dark) cycles at $24 \pm 2{ }^{\circ} \mathrm{C}$ for at least 7 days. Data are expressed as the mean of $n=3$ biological replicates \pm SEM (standard error of the mean). White and black bars represent light and dark phases, respectively. Relative expression was normalized using elongation factor 1 (NtEFl) as housekeeping gene. Black and grey lines represent HRC treated with water (control condition) and phenol (100 $\mathrm{mg} / \mathrm{L}$ during $24 \mathrm{~h}$ ), respectively AQ3
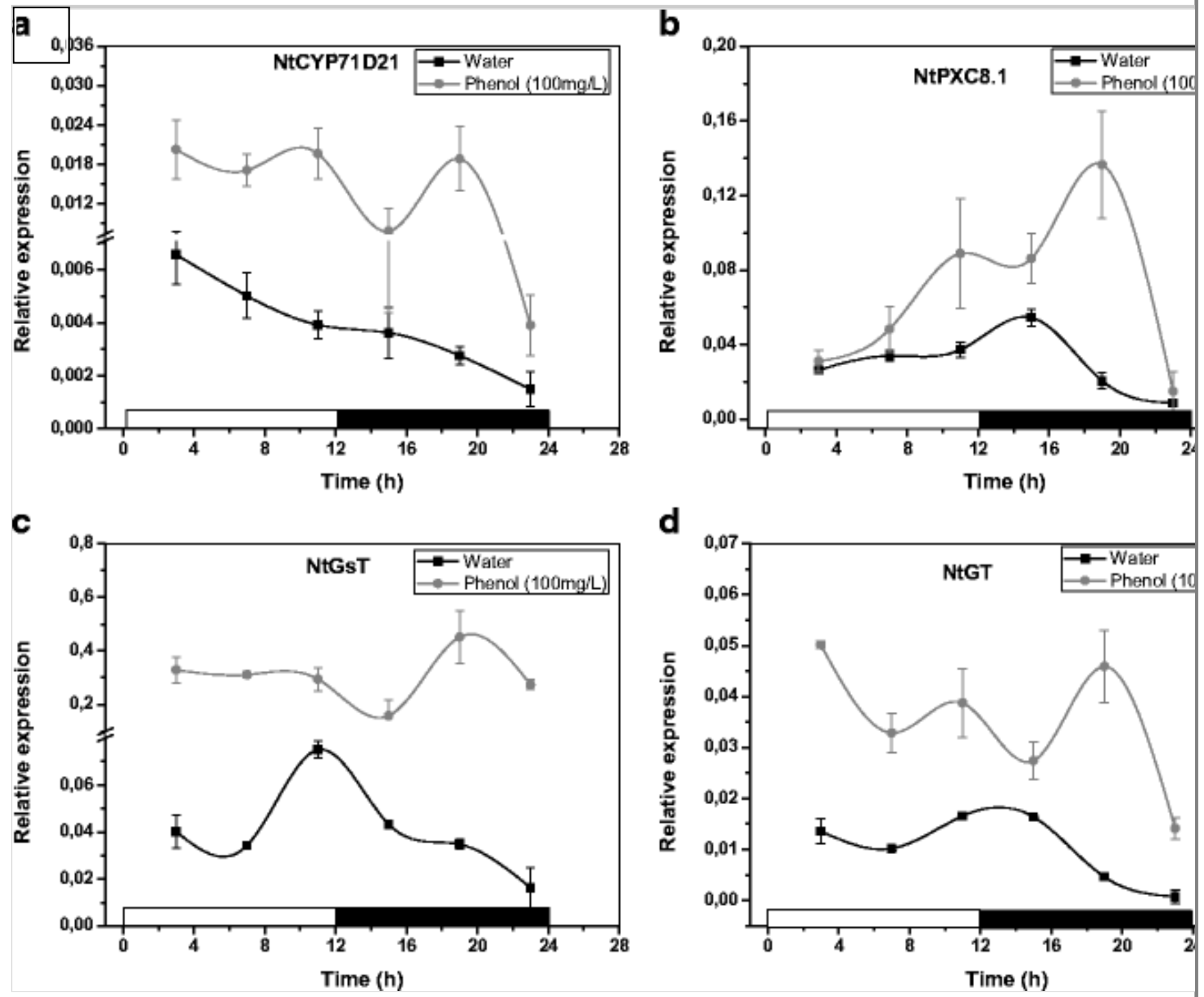

Following phenol treatment, all genes examined were significantly upregulated in both 2- and 3-week-old HRC. In 2-week-old HRC, phenol exposure led to a rhythmic oscillatory pattern of NtCYP71D21 and NtPXC8.1 that was anti-phasic to the oscillations found under control conditions (Fig. 5a, b). Although the oscillations of $N t G s T$ and $N t G T$ were not very rhythmic under control 
conditions, treatment with phenol revealed a trend consisting of a downregulation during the day and upregulation during the night (Fig. 5c, d). In 3-week-old HRC, phenol treatment not only induced a significant upregulation in the expression of all evaluated genes but also a loss of their rhythmic profiles (Fig. 6a-d). Similar results were found in homolog tobacco MXC-related genes identified in Arabidopsis seedlings which were also upregulated after exposure to phenol and acid-extractable organic fraction (Xu et al. 2012; Widdup et al. 2015). Based in our results, we argue that phenol treatment would work as an alternative regulatory signal masking the circadian regulation. This alternative regulation might also represent the difference between an acute response to high phenol levels versus lower levels where circadian regulation might be more sufficient/appropriate for regulating detoxification. However, it is important to note that the temporal expression of the MXC genes in response to phenol treatment was much more consistent and robust in 2-week-old HRC (Fig. 5) than in 3-week-old HRC (Fig. 6). Given a lack of rhythms observed in clock gene expression under DD in 3-week-old HRC (Fig. S3b), which suggests that the clock may be less robust in HRC of this age compared to 2-week-old HRC, it would be supposed that an impaired clock might contribute to the apparent weaker temporal regulation of MXC-related genes in 3-week-old HRC. Consistent with the rhythmic oscillatory pattern found in our work, a search for circadian cis-regulatory elements in the putative genes related to MXC revealed the presence of at least one circadian cis-regulatory sequences (CCRS) of 10-11 nucleotides with the following consensus sequence CAANNNNATC (where N corresponds to any nucleotides, Supporting information Table S3). These sequences would be important for the circadian regulation of their mRNA accumulation. However, deletion analysis or reporter studies would be required to demonstrate that these putative cis-regulatory sequences are involved in the daily fluctuations of MXC-related gene expression. In addition, homologs of tobacco MXC-related genes identified in Arabidopsis (Supporting information Table S4) also revealed the presence of at least one CCRS of 8-9 nucleotides in their promoter sequences (Supporting information Table S5), suggesting that expression of these Arabidopsis genes could also be under a circadian control.

The results of our studies show that the putative genes coding for enzymes involved in xenobiotic metabolism display rhythmic expression profiles that are differentially modulated after phenol treatment. These results resemble studies in animal systems in which the expression of genes involved in detoxification pathways, mainly in the liver, are under a circadian control (Zmrzljak and Rozman 2012). Our studies thus provide new evidences of some similarities between the plant and animal systems in the circadian regulation of xenobiotic metabolism. 


\section{Conclusions}

This work described the presence of some putative key genes of the molecular circadian clock in $N$. tabacum, which shown oscillatory expression profiles. These oscillatory clock genes include NtLHY, NtTOC1, NtFKF1, NtGI and NtPRR9 and are present in both seedlings and HRC grown under in vitro conditions and synchronized by LD cycles (Figs. 1, 2, and 3). We found that not only seedlings but also HRC sustain diurnal expression of these clock gene orthologs (Figs. 2 and 3). The observed oscillation in HRC opens the possibility that root cultures conserve photosensitive components (including photoreceptors) capable of perceiving light/dark changes essential to synchronize the rhythmic expression of clock components (Bordage et al. 2016). Under constant darkness, some clock genes (NtLHY, NtTOC1 and NtFKF1) seem to lose their rhythmic expression mainly in 3-week-old HRC (Fig. S3b). This result supports the crucial role of light as synchronizer and suggests that the age of HRC may have an important role in the performance of clock gene expression and therefore in the response to phenol treatment. Recent studies performed with Arabidopsis, showed that not only the light but also metabolic signals can play an important role in entraining the clock (Dodd et al. 2015). Our studies also show that, in HRC, phenol treatment downregulates the expression of most clock genes examined with the exception of NtFKF1 and NtGI that were significantly upregulated in 2-week-old HRC (Fig. 2c, d). Furthermore, in both seedlings and $\mathrm{HRC}$, the expression of four putative genes involved in MXC follows a rhythmic pattern that might be controlled through a circadian cis-regulatory motif present in the promoters of these putative MXC genes (Supporting information Table S3). Changes in the circadian expression of clock genes might be also correlated with the upregulated or anti-phasic expression profiles observed in MXC genes following phenol treatment (Figs. 5 and 6).

Altogether, our studies show the usefulness of HRC as a model system to study circadian behavior and the possible role of the circadian clock in the control of MXC gene expression. Further analyses will help us improve our understanding of phenol metabolism in plants and the importance of the circadian clock for proper responses to xenobiotic stress.

\section{Acknowledgements}

L.S.A, M.E.G and E.A. are members of the research career of Consejo Nacional de Investigaciones Científicas y Técnicas (CONICET), Argentina. L.S.A had a fellowship (Partial Financing Program for Short Stays Abroad) from CONICET. This work was supported by grants from CONICET, ANPCyT and SECyTUNRC. This work was also supported by research grants to P.M. from the Spanish Ministry of Economy and Competitiveness, from the Generalitat de 
Catalunya (AGAUR), from the Global Research Network of the National Research Foundation of Korea, from the European Commission Marie Curie Research Training Network (ChIP-ET) and from the Spanish Ministry of Economy and Competitiveness, through the "Severo Ochoa Programme for Centres of Excellence in R\&D” 2016-2019 (SEV-2015-0533)".

\section{Electronic supplementary material}

\section{ESM 1}

(DOCX $149 \mathrm{~kb}$ )

\section{References}

Abhilash PC, Jamil S, Singh N (2009) Transgenic plants for enhanced biodegradation and phytoremediation of organic xenobiotics. Biotechnol Adv 27:474-488. https://doi.org/10.1016/j.biotechadv.2009.04.002

Agostini E, Coniglio MS, Milrad SR et al (2003) Phytoremediation of 2,4dichlorophenol by Brassica napus hairy root cultures. Biotechnol Appl Biochem 37:139-144. doi:10.1042/BA20020079

Agostini E, Talano MA, González PS et al (2013) Application of hairy roots for phytoremediation: what makes them an interesting tool for this purpose? Appl Microbiol Biotechnol 97:1017-1030

Alabadi D, Oyama T, Yanovsky MJ et al (2001) Reciprocal regulation between TOC1 and LHY/CCA1 within the Arabidopsis circadian clock. Science (New York, NY) 293:880-883.

https://doi.org/10.1126/science. 1061320

Alabadi D, Yanovsky MJ, Mas P et al (2002) Critical role for CCA1 and LHY in maintaining circadian rhythmicity in Arabidopsis. Current biology : CB $12: 757-761$

Bordage S, Sullivan S, Laird J et al (2016) Organ specificity in the plant circadian system is explained by different light inputs to the shoot and root 
clocks. New Phytol 212:136-149. https://doi.org/10.1111/nph.14024

Coniglio MS, Busto VD, González PS et al (2008) Application of Brassica napus hairy root cultures for phenol removal from aqueous solutions. Chemosphere 72:1035-1042. https://doi.org/10.1016/j.chemosphere.2008.04.003

Dodd AN, Belbin FE, Frank A, Webb AAR (2015) Interactions between circadian clocks and photosynthesis for the temporal and spatial coordination of metabolism. Front Plant Sci 6:245. https://doi.org/10.3389/fpls.2015.00245

Dodd AN, Salathia N, Hall A et al (2005) Plant circadian clocks increase photosynthesis, growth, survival, and competitive advantage. Science (New York, NY) 309:630-633. https://doi.org/10.1126/science.1115581

Dong MA, Farre EM, Thomashow MF (2011) CIRCADIAN CLOCKASSOCIATED 1 and LATE ELONGATED HYPOCOTYL regulate expression of the C-REPEAT BINDING FACTOR (CBF) pathway in Arabidopsis. Proc Natl Acad Sci 108:7241-7246.

https://doi.org/10.1073/pnas.1103741108

Farre EM, Harmer SL, Harmon FG et al (2005) Overlapping and distinct roles of PRR7 and PRR9 in the Arabidopsis circadian clock. Current biology : CB 15:47-54. https://doi.org/10.1016/j.cub.2004.12.067

Fraissinet-Tachet L, Baltz R, Chong J et al (1998) Two tobacco genes induced by infection, elicitor and salicylic acid encode glucosyltransferases acting on phenylpropanoids and benzoic acid derivatives, including salicylic acid. FEBS Lett 437:319-323. https://doi.org/10.1016/S0014-5793(98)01257-5

Habte E, Müller LM, Shtaya M et al (2014) Osmotic stress at the barley root affects expression of circadian clock genes in the shoot. Plant, Cell and Environment 37:1321-1337. https://doi.org/10.1111/pce.12242

Harb A, Krishnan A, Ambavaram MMR, Pereira A (2010) Molecular and physiological analysis of drought stress in Arabidopsis reveals early responses leading to acclimation in plant growth. Plant Physiol 154:12541271. https://doi.org/10.1104/pp.110.161752

Harmer SL, Kay SA (2005) Positive and negative factors confer phasespecific circadian regulation of transcription in Arabidopsis. Plant Cell 17:1926-1940. https://doi.org/10.1105/tpc.105.033035 
Ibáñez SG, Alderete LGS, Medina MI, Agostini E (2012) Phytoremediation of phenol using Vicia sativa L. plants and its antioxidative response. Environ Sci Pollut Res 19:1555-1562. https://doi.org/10.1007/s11356-011-0664-4

Kant P, Gordon M, Kant S et al (2008) Functional-genomics-based identification of genes that regulate Arabidopsis responses to multiple abiotic stresses. Plant, Cell and Environment 31:697-714. https://doi.org/10.1111/j.1365-3040.2008.01779.x

Lanoue A, Shakourzadeh K, Marison I et al (2004) Occurrence of circadian rhythms in hairy root cultures grown under controlled conditions. Biotechnol Bioeng 88:722-729. https://doi.org/10.1002/bit.20268

Lescot M, Dehais P, Thijs G et al (2002) PlantCARE, a database of plant cisacting regulatory elements and a portal to tools for in silico analysis of promoter sequences. Nucleic Acids Res 30:325-327

Lu SX, Knowles SM, Andronis C, et al (2009) CIRCADIAN CLOCK ASSOCIATED1 and LATE ELONGATED HYPOCOTYL function synergistically in the circadian clock of Arabidopsis. Plant Physiol 150:834-843. https://doi.org/10.1104/pp.108.133272

Marcolino-Gomes J, Rodrigues FA, Fuganti-Pagliarini R et al (2014) Diurnal oscillations of soybean circadian clock and drought responsive genes. PLoS One. https://doi.org/10.1371/journal.pone.0086402

Más P (2008) Circadian clock function in Arabidopsis thaliana: time beyond transcription. Trends Cell Biol 18:273-281

Mas P, Kim W-Y, Somers DE, Kay SA (2003) Targeted degradation of TOC1 by ZTL modulates circadian function in Arabidopsis thaliana. Nature 426:567-570. https://doi.org/10.1038/nature02163

McClung RC (2006) Plant circadian rhythms. Plant Cell 18:792-803. https://doi.org/10.1017/CBO9781107415324.004

Mishra P, Panigrahi KC (2015) GIGANTEA - an emerging story. Front Plant Sci 6:8. https://doi.org/10.3389/fpls.2015.00008

Murashige T, Skoog F (1962) A revised medium for rapid growth and bioassays with tobacco tissue cultures. Physiol Plant 15:473-497. 
https://doi.org/10.1111/j.1399-3054.1962.tb08052.x

Nagel D, Kay S (2012) Complexity in the wiring and regulation of plant circadian networks. Curr Biol 22:R648-R657.

https://doi.org/10.1016/j.cub.2012.07.025

Nakamichi N, Kita M, Niinuma K et al (2007) Arabidopsis clock-associated pseudo-response regulators PRR9, PRR7 and PRR5 coordinately and positively regulate flowering time through the canonical CONSTANSdependent photoperiodic pathway. Plant \& cell physiology 48:822-832. https://doi.org/10.1093/pcp/pcm056

Ralston L, Kwon ST, Schoenbeck M et al (2001) Cloning, heterologous expression, and functional characterization of 5-epi-aristolochene-1,3dihydroxylase from tobacco (Nicotiana tabacum). Arch Biochem Biophys 393:222-235. https://doi.org/10.1006/abbi.2001.2483

Salomé PA, McClung CR (2005) PSEUDO-RESPONSE REGULATOR 7 and 9 are partially redundant genes essential for the temperature responsiveness of the Arabidopsis circadian clock. Plant Cell 17:791-803. https://doi.org/10.1105/tpc.104.029504.In

Sanchez A, Shin J, Davis SJ (2011) Abiotic stress and the plant circadian clock. Plant Signal Behav 6:223-231. https://doi.org/10.4161/psb.6.2.14893

Sawa M, Nusinow DA, Kay SA, Imaizumi T (2007) FKF1 and GIGANTEA complex formation is required for day-length measurement in Arabidopsis. Science (New York, NY) 318:261-265. https://doi.org/10.1126/science.1146994

Schmidt GW, Delaney SK (2010) Stable internal reference genes for normalization of real-time RT-PCR in tobacco (Nicotiana tabacum) during development and abiotic stress. Mol Gen Genomics 283:233-241. https://doi.org/10.1007/s00438-010-0511-1

Seo PJ, Mas P (2015) STRESSing the role of the plant circadian clock. Trends Plant Sci:1-8. https://doi.org/10.1016/j.tplants.2015.01.001

Song YH, Estrada DA, Johnson RS et al (2014) Distinct roles of FKF1, Gigantea, and Zeitlupe proteins in the regulation of CONSTANS stability in Arabidopsis photoperiodic flowering. Proc Natl Acad Sci U S A 111:1767217677. https://doi.org/10.1073/pnas.1415375111 
Sosa Alderete LG, Agostini E, Medina MI (2011) Antioxidant response of tobacco (Nicotiana tabacum) hairy roots after phenol treatment. Plant Physiol Biochem 49:1020-1028. https://doi.org/10.1016/j.plaphy.2011.07.009

Sosa Alderete LG, Talano MA, Ib????ez SG et al (2009) Establishment of transgenic tobacco hairy roots expressing basic peroxidases and its application for phenol removal. J Biotechnol 139:273-279. https://doi.org/10.1016/j.jbiotec.2008.11.008

Takahashi Y, Nagata T (1992) parB: an auxin-regulated gene encoding glutathione S-transferase. Proc Natl Acad Sci U S A 89:56-59

Talano MA, Frontera S, González P et al (2010) Removal of 2,4diclorophenol from aqueous solutions using tobacco hairy root cultures. J Hazard Mater 176:784-791. https://doi.org/10.1016/j.jhazmat.2009.11.103

Vaze KM, Sharma VK (2013) On the adaptive significance of circadian clocks for their owners. Chronobiol Int 30:413-433.

https://doi.org/10.3109/07420528.2012.754457

Widdup EE, Chatfield-Reed K, Henry D et al (2015) Identification of detoxification pathways in plants that are regulated in response to treatment with organic compounds isolated from oil sands process-affected water. Chemosphere 139:47-53. https://doi.org/10.1016/j.chemosphere.2015.05.048

Xu J, ZH S, Chen C et al (2012) Stress responses to phenol in Arabidopsis and transcriptional changes revealed by microarray analysis. Planta 235:399410. https://doi.org/10.1007/s00425-011-1498-5

Yakir E, Hilman D, Kron I et al (2009) Posttranslational regulation of CIRCADIAN CLOCK ASSOCIATED1 in the circadian oscillator of Arabidopsis. Plant Physiol 150:844-857.

https://doi.org/10.1104/pp.109.137414

Yon F, Seo P-J, Ryu JY et al (2012) Identification and characterization of circadian clock genes in a native tobacco, Nicotiana attenuata. BMC Plant Biol 12:172. https://doi.org/10.1186/1471-2229-12-172

Zmrzljak UP, Rozman D (2012) Circadian regulation of the hepatic endobiotic and xenobitoic detoxification pathways: the time matters. Chem Res Toxicol 25:811-824. https://doi.org/10.1021/tx200538r 
Burken JG, Uktake and metabolism of organic compounds: Green-liver model. In: Phytoremediation: Transformation and control of contaminants, McCutcheon SC, Schnoor JL (eds). Wiley Interscience. John Wiley and Sons, Inc, Hoboken, New Jersey, 2003:59-84. 